\title{
Malignant transformation in a sciatic plexiform neurofibroma in Neurofibromatosis Type 1 - imaging features that aid diagnosis
}

\author{
Dorinda Chee Yee Chew ${ }^{1 *}$, Daniel Bin Hang Zhao ${ }^{1}$, Kesavan Sittampalam $^{2}$, S Krishna Kumar ${ }^{1}$ \\ 1. Department of Oncology Imaging, National Cancer Centre, Singapore \\ 2. Department of Pathology, National Cancer Centre, Singapore

\begin{abstract}
* Correspondence: Chew Chee Yee Dorinda, Department of Oncology Imaging, National Cancer Centre, 11 Hospital Drive, Singapore 169610, Singapore

$(\checkmark$ dorinda.chew@mohh.com.sg)
\end{abstract}

Radiology Case. 2020 Dec; 14(12):1-13 :: $\quad$ DOI: 10.3941/jrcr.v14i12.4028

\begin{abstract}
A 41-year-old Asian male with NF1 and bilateral sciatic plexiform neurofibromas, presented with unintentional weight loss, increasing size of a left thigh mass associated with increasing pain and radiculopathy. MRI of the left thigh demonstrated imaging features suspicious of malignant transformation. The patient had a new left lung mass, demonstrating avid FDG uptake, raising suspicion for metastasis. Surgical resection of the left thigh mass confirms malignant transformation in a preexisting sciatic plexiform neurofibroma. Diagnosis of malignant transformation in a nerve sheath tumour can be challenging. MRI remains the main preferred imaging modality in the evaluation of these tumours. Imaging features that raise suspicion for malignant transformation are discussed. Although none of these are specific for malignant transformation, studies suggest that the presence of two to four of these features should prompt further investigations.
\end{abstract}

\section{CASE REPORT}

\section{CASE REPORT}

\section{Clinical History}

A 41-year-old Asian male presented with low-grade fever and unintentional loss of weight of $5 \mathrm{~kg}$ over 5 -6months. He also complained of increasing size of his left thigh, associated with increasing pain and radiculopathy.

The patient is a known case of Neurofibromatosis type 1 (NF1). He is also known to have bilateral sciatic plexiform neurofibromas. Clinical examination revealed multiple cutaneous neurofibromas and café-au-lait spots on his body and limbs. Clinically palpable soft tissue masses were felt in the bilateral posterior thighs, measuring up to $21 \times 27 \mathrm{~cm}$ on the left, and $16 \times 18 \mathrm{~cm}$ on the right. No obvious skin changes were detected.

\section{Imaging findings}

Bilateral lower limb radiographs demonstrated large posterior soft tissue masses in both thighs with no evidence of bony erosion, periosteal reaction, or pathological fracture (Figure 1). 
Magnetic Resonance Imaging (MRI) of the left thigh demonstrated an $11 \times 11 \times 32 \mathrm{~cm}$, heterogeneous soft tissue mass seen in the posterior compartment, with tumoural heterogeneous signal and peripheral rim enhancement. Perilesional oedema was seen in the adjacent soft tissues. Superiorly, the mass was contiguous, but distinct, from a lumbosacral plexus mass. Findings raised suspicion for malignant transformation of a preexisting plexiform neurofibromatosis of the lumbosacral plexus. Subcutaneous/cutaneous soft tissue nodules demonstrating homogeneous T2 signal and enhancement were noted, in keeping with benign neurofibromas (Figure 2).

MRI of the right thigh revealed multiple peripheral nerve sheath tumours along the course of the right sciatic nerve, as well as the obturator and proximal right femoral nerves. These lesions demonstrated predominantly $\mathrm{T} 2 \mathrm{w}$ hyperintense cystic signal with central enhancement. The dominant lesion located in the mid-distal posterior thigh, measured $10.1 \mathrm{~cm}$ in longest dimension and demonstrated thin peripheral enhancement, suggestive for possible malignant transformation (Figure 3).

A chest radiograph performed revealed a new left paramediastinal mass. Subsequent staging Positron Emission Tomography/Computed Tomography (PET/CT) study performed confirmed that the new $6.2 \times 5.1 \times 6.4 \mathrm{~cm}$ left paramediastinal lesion demonstrates avid fluorodeoxyglucose (FDG) tracer activity (SUV max 24.2), raising suspicion for metastasis (Figure 4).

Multiple, benign bilateral pleural based soft tissue opacities were also seen on the chest radiograph, some associated with rib scalloping, in keeping with neurofibromata (Figure 5).

Several hypodense lesions were also seen occupying the deep spaces of the neck bilaterally. Of note, one was seen arising from the right carotid space, as evidenced by splaying of the right external and internal carotid arteries. This mass demonstrated heterogeneous enhancement, with a solid component showing moderate FDG activity (SUV max 6.3) worrisome for malignant transformation (Figure 6). A decision was made to follow this with serial imaging.

Multiple paravertebral masses were also identified. A T11-12 left paravertebral soft tissue lesion was seen demonstrating characteristic "dumb-bell" shape, with widening of the left neural foramen and compression of the spinal cord (Figure 7). In addition, presacral soft tissue masses were also noted, expanding the sacral neural foramina, and extending to involve the bilateral lumbosacral plexus, consistent with plexiform neurofibromas (Figure 8).

An MRI of the brain demonstrated no evidence of intracranial metastases. Incidentally, a left basifrontal encephalocele was seen, secondary to dysplasia of the left basifrontal skull (Figure 9).

\section{Management and Follow-up}

The patient underwent surgical excision of the left thigh posterior compartment tumour. His post-operative course was uncomplicated. He is currently being followed-up by a multidisciplinary team and is receiving ongoing chemotherapy and radiotherapy. The right carotid body tumour and dominant right posterior thigh mass are being monitored by serial imaging.

Histology of the left posterior thigh compartment tumour demonstrated a grade 3 malignant peripheral nerve sheath tumour (MPNST) arising from a pre-existing plexiform neurofibroma, with areas of tumoral necrosis and hemorrhage. The margins are negative of tumour involvement and there is no evidence of lymphovascular or perineural invasion. The tumour stains positive for S100, CD34 and H3K27me3 (Figure 10).

\section{DISCUSSION}

\section{Etiology \& Demographics:}

NF1, also known as von Recklinghausen disease, is the most common type of neurocutaneous syndrome. Its incidence is approximately 1 in 2500 to 3000 with no gender predilection. It is inherited in an autosomal dominant fashion or secondary to spontaneous mutation of the NF1 gene, which is located on chromosome 17. There is resultant decreased production of neurofibromin, which is a tumour suppressor. As such, NF1 patients are at significantly increased risk for developing both benign and malignant tumours.

NF1 is a multisystem disease, with significant variability between individuals. The diagnostic criteria include the presence of 2 or more of the following: first degree relative with NF1, 6 or more café-au-lait spots, 2 or more neurofibromas or 1 plexiform neurofibroma, optic nerve glioma, bony dysplasia, axillary or inguinal freckling and 2 or more Lisch nodules.

MPNSTs are relatively rare, accounting for 5-10\% of all adult soft tissue sarcomas, with $25-50 \%$ of cases associated with NF1. It is estimated to occur at a rate of $0.001 \%$ in the general population, but this increases to $8-13 \%$ amongst NF1 patients [1]. It may arise from any pre-existing benign peripheral nerve sheath tumour (BPNST) but is 20 times more likely to arise from a plexiform neurofibroma [2-4]. A plexiform neurofibroma is a neurofibroma that grows along the length of a nerve and its branches.

Patients with NF1 who develop malignant transformation, tend to be of younger age with larger tumours. They also tend to present later in their disease, probably due to overlapping clinical symptoms amongst the benign tumours they already have. Prognostic factors include NF1, gross tumour resectability, tumour size, tumour location and surgical margin status [5]. 
As there are currently no guidelines for screening for MPNSTs, clinicians and radiologists should be familiar with symptoms and imaging features suggestive of malignant transformation.

\section{Clinical \& Imaging findings:}

High risk clinical symptoms include acute onset of discomfort or pain, resting pain, new neurologic symptoms, increase in size of the mass in adults or change in consistency from soft to hard. Diagnosis remains difficult due to overlaps in clinical symptoms also seen in preexisting BPNSTs.

Radiographs are of limited value except in the evaluation of potential aggressive bony features (e.g. periosteal reaction, bony erosion), which may help direct dedicated imaging. Ultrasonography (US) is easily accessible and cost effective, however it is also of limited value in evaluating deeply located lesions. Computed Tomography (CT) is effective in delineating the extent of the tumour, identify bony involvement and good for preoperative planning. FDG PET shows promise as a useful adjunct to help distinguish between benign and malignant neurofibromas, plays a role in staging and directs biopsy to the highest grade of tumour.

MRI remains the best imaging modality for evaluating MPNSTs due to its high soft tissue resolution and ability to further characterize different components of the tumour and delineate margins for surgical planning. Imaging features suggestive of malignant transformation include an enlarging mass, presence of peripheral enhancement, perilesional oedema, and increased tumour heterogeneity.

\section{Increased largest dimension of mass}

Interval increase in the largest dimension of a known peripheral nerve sheath tumour, especially if associated with high risk clinical manifestations such as pain at rest, increasing or acute onset of pain, neurologic symptoms and radiculopathy, should raise suspicion for malignant transformation $[3,6]$. The interval increase in size is probably due to rapid growth in malignant tumour. The average size of a MPNST is usually above $5 \mathrm{~cm} \mathrm{[3].}$

\section{Increased tumour heterogeneity}

Malignant transformation of a tumour can result in hemorrhage or necrosis, which is reflected as heterogeneity on T1-weighted images, and intra-tumoral cystic change on T2weighted images. Some studies reported that a 'target sign' may be helpful in differentiating between benign and malignant neurofibromas. A target sign is described as a central hypointense region seen on T2-weighted images, surrounded by a rim of $\mathrm{T} 2$ hyperintensity. This sign has been commonly associated with localized benign neurofibroma, corresponding to a central area of hypercellular stroma surrounded by myxomatous tissue on histopathology [7]. Loss of the target sign may be suggestive of malignant transformation, although this sign is not sensitive, as not all benign neurofibromas exhibit this imaging feature $[3,6-8]$.

\section{Presence of peripheral enhancement}

Ogose et al [9] reported development of peripheral enhancement, as opposed to focal central enhancement, a sign that is often observed in benign neurogenic tumours, should raise suspicion for malignant transformation. Although, other peripheral nerve sheath tumours such as Schwannomas can demonstrate peripheral enhancement because of cystic change, this feature is rare in neurofibromas. Yu et al [3] suggest that the presence of peripheral enhancement, accompanied by increased tumour heterogeneity (rather than pure cystic change) may be valuable in diagnosis.

\section{Peri-lesional oedema}

BPNSTs tend to be more well-defined and are usually surrounded by a capsule. Peri-lesional oedema occurs when malignant transformation of an existing peripheral nerve sheath tumour occurs with marginal infiltration of tumour cells into surrounding soft tissue, causing a localized reaction. However, this lacks specificity, as well-defined tumour margins were also seen in MPNSTs [6].

Patel et al [2] suggests that the presence of two to four of these features is suggestive of malignancy with a specificity of $90 \%$ and sensitivity of $61 \%$. As such, if a tumour is highly suspicious, a biopsy is recommended for early diagnosis.

\section{Treatment \& Prognosis:}

Most MPNSTs are aggressive, high grade sarcomas with a high likelihood of local recurrence (40-65\%) and distant metastases. They frequently metastasize to the lungs, followed by the bones, brain and liver. MPNSTs have a poor outcome if untreated. Complete surgical excision continues to be the gold standard of treatment, with adjuvant chemotherapy and radiotherapy administered in advanced disease. However, it remains to have a relatively poor prognosis with a disease specific mortality at 10 years after definitive surgery of $43 \%$ [5].

\section{Differential Diagnoses:}

Given the constellation of findings, the diagnosis in this case is pathognomonic of NF1. Malignant transformation in pre-existing BPNST needs to be excluded. Other differential diagnoses (Table 2) of an aggressive soft tissue mass will include other types of soft tissue sarcomas [10,11].

\section{TEACHING POINT}

Differentiating malignant transformation of benign tumours continues to be a challenge in clinical practice with radiology playing an important role in answering clinical questions; MRI continues to be the modality of choice for characterizing and delineating the target lesion while CT and PET-CT have been useful for staging disseminated disease, with the latter also helpful in identifying the best location for image guided biopsy. Imaging features of malignant transformation guide radiologists in sieving sinister lesions from benign entities; high risk factors would include: increased largest dimension of mass, increased tumour heterogeneity, peripheral enhancement and perilesional oedema, as in our case study. 


\section{REFERENCES}

1. Park ER, Smith KB, Merker VL et al. Examining perceived cancer risk among patients with neurofibromatosis type 1 . Journal of Neuro-Oncology 2015; 122:127. PMID 25559686

2. Patel NB and Stacy GS. Musculoskeletal Manifestations of Neurofibromatosis Type 1. American Journal of Roentgenology 2012; 199:W99. PMID: 22733937

3. Yu YH, Wu JT, Ye J, Chen MX. Radiological findings of malignant peripheral nerve sheath tumour: reports of six cases and review of literature. World Journal of Surgical Oncology 2016; 14:142. PMID 27159980

4. Tucker T, Wolkenstein P, Revuz J, Zeller J, Friedman JM. Association between benign and malignant peripheral nerve sheath tumours in NF1. Neurology 2005; 65:205. PMID: 16043787

5. Anghileri M, Miceli R, Fiore $\mathrm{M}$ et al. Malignant peripheral nerve sheath tumours: prognostic factors and survival in a series of patients treated at a single institution. Cancer 2006; 107:1065. PMID 16881077

6. Wasa J, Nishida Y, Tsukushi S et al. MRI features in the Differentiation of Malignant Peripheral Nerve Sheath Tumors and Neurofibromas. American Journal of Roentgenology 2010; 194:1568. PMID: 20489098

7. Bhargava R, Parham DM, Lasater OE, Chari RS, Chen G, Fletcher BD. MR imaging differentiation of benign and malignant peripheral nerve sheath tumours: use of the target sign. Pediatric Radiology 1997; 27:124. PMID: 9028843

8. Fortman BF, Kuszyk BS, Urban BA, Fishman EK. Neurofibromatosis Type 1: A Diagnostic Mimicker at CT. Radiographics 2001; 21:601-612. PMID: 11353109

9. Ogose A, Hotta T, Morita T et al. Tumours of peripheral nerves: correlation of symptoms, clinical signs, imaging features, and histologic diagnosis. Skeletal Radiology 1999; 28:183. PMID: 10384987

10. Baheti AD, Malley RBO, Kim $S$ et al. Soft-Tissue Sarcomas: An update for radiologists based on the revised 2013 World Health Organisation classification. American Journal of Roentgenology 2016; 206:924. PMID: 26998884

11. Aga P, Singh R, Parihar A, Parashari U. Imaging Spectrum in Soft Tissue Sarcomas. Indian Journal of Surgical Oncology 2011; 2(4):271. PMID: 23204782

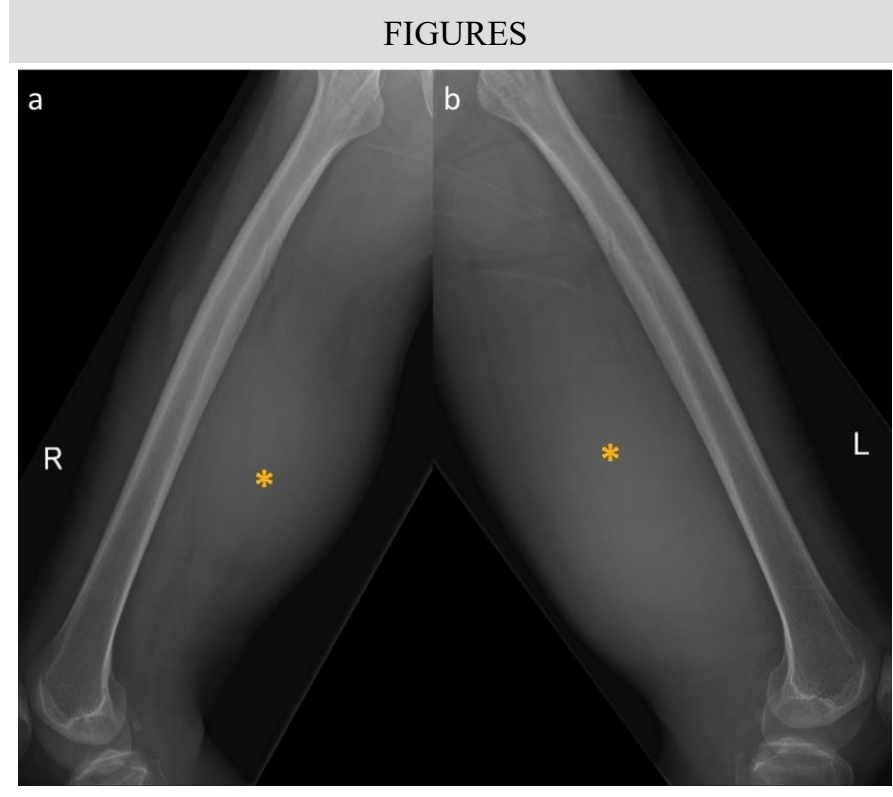

Figure 1: 41-year-old male with NF1. Bilateral posterior thigh soft tissue masses noted, larger on the left.

Findings: (a) Right femoral radiograph in lateral projection and (b) left femoral radiograph in lateral projection demonstrating bilateral posterior thigh soft tissue masses $(*)$, larger on the left. No bony erosion, periosteal reaction or pathological fracture.

Technique: (a) Right femoral radiograph in lateral projection (kVp: 73 and mAs: 8) and (b) left femoral radiograph in lateral projection (kVp: 73 and mAs: 8). Siemens Fluorospot Compact FD. 


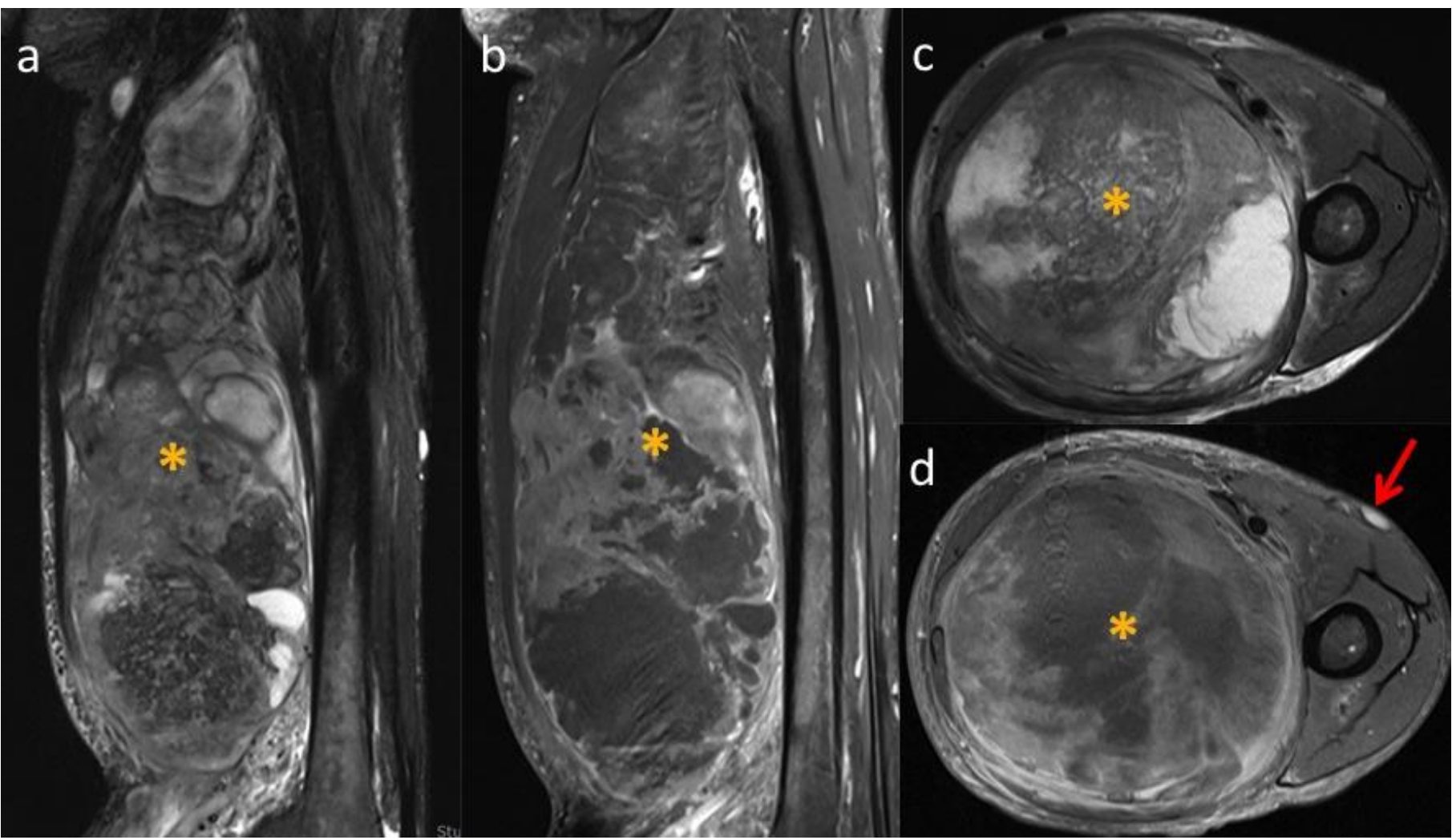

Figure 2: 41-year-old male with NF1. A left posterior thigh sciatic plexiform neurofibroma with MRI features suspicious for malignant transformation.

Findings: ( $a$ and b) Sagittal and (c and d) Axial contrast-enhanced MRI of the left thigh demonstrating a large posterior thigh mass, measuring up to $32 \mathrm{~cm}$ in longest dimension, with intra-tumoral heterogeneity and heterogeneous enhancement. It demonstrates thick peripheral enhancement with peri-lesional oedema noted along the inferior aspect of the tumour $(*)$. Findings are suspicious for a sciatic nerve plexiform neurofibroma with malignant transformation. There is displacement and atrophy of the adjacent muscle. No abnormal marrow signal detected. (d) A T2w hyperintense, homogeneously enhancing subcutaneous nodule seen in the left medial thigh is in keeping with a known benign neurofibroma (red arrow).

Technique: (a-d) MRI contrast enhanced left thigh, Siemens Skyra 3T. Sagittal T2 TIRM (TR: 5670.00, TI: 200.00, TE: 69.00, SW: 4.40mm), Sagittal T1 FS post contrast (TR: 509.00, TE: 8.40, SW: 4.40mm), Axial T2 TSE TRA FS (TR: 5760.00, TE: 67.00, SW: $5.00 \mathrm{~mm}$ ) and Axial T1 TSE TRA FS post contrast (TR: 519.00, TE: 11.00, SW: 5.00mm). Intravenous contrast: 10ml Dotarem. 


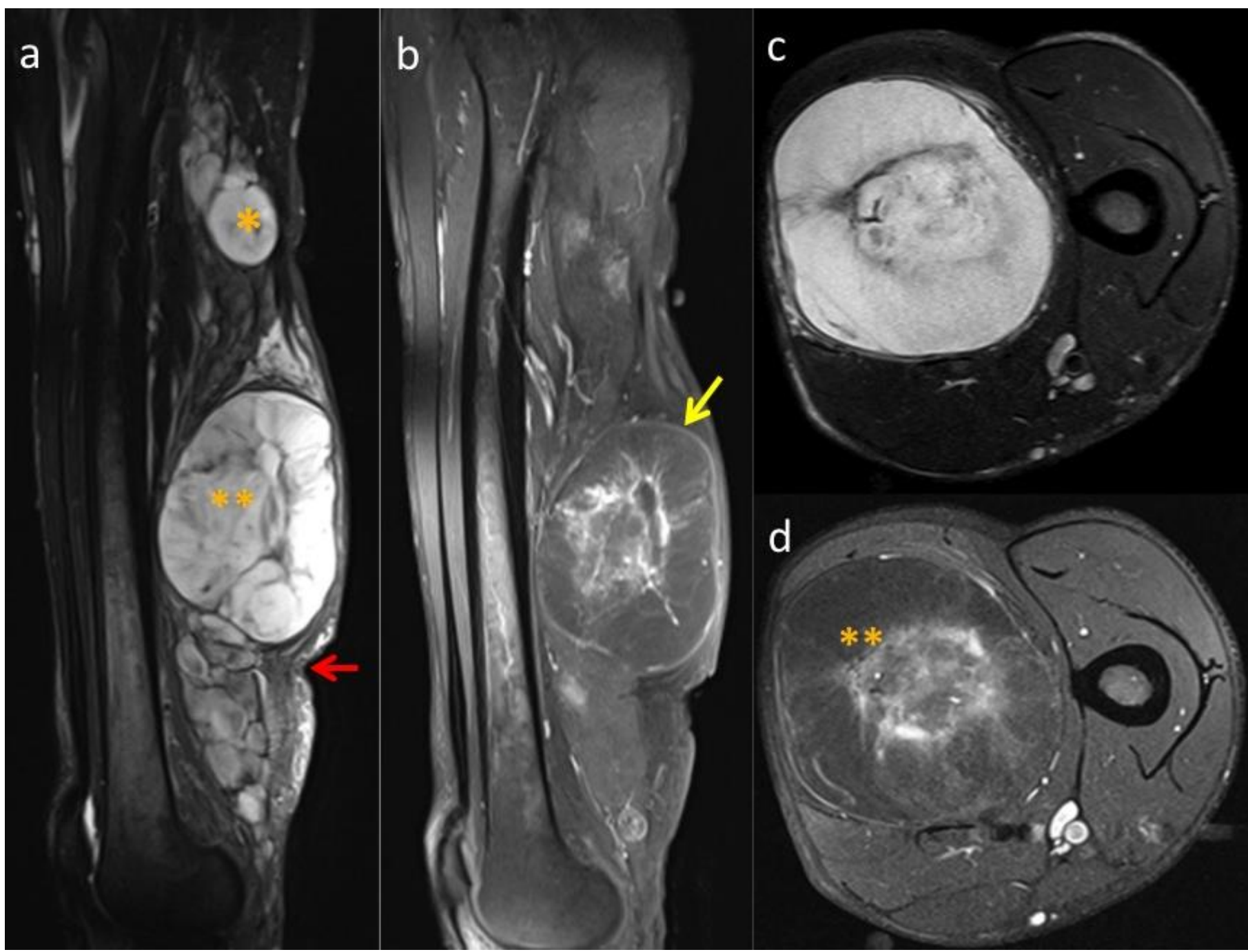

Figure 3: 41-year-old male with NF1. Right posterior thigh sciatic plexiform neurofibromas. The dominant mass demonstrate MRI features indeterminate for malignant transformation.

Findings: (a-b) Sagittal and (c-d) Axial contrast-enhanced MRI of the right thigh demonstrating several posterior thigh masses (*) along the course of the sciatic nerve, with majority demonstrating predominant $\mathrm{T} 2 \mathrm{w}$ hyperintense signal and central enhancement. The dominant lesion located in the mid-distal thigh, measures up to $10.1 \mathrm{~cm}$ in largest dimension, demonstrates mild heterogeneous T2 signal, predominant central enhancement (**) and a thin peripheral rim of enhancement (yellow arrow). Mild adjacent peri-lesional oedema is also seen (red arrow). Findings are indeterminate of malignant transformation. No abnormal marrow signal.

Technique: (a-d) MRI contrast enhanced right thigh, Siemens Avanto 1.5T. Sagittal T2 TIRM (TR: 3270.00, TI: 150.00, TE: 70.00, SW: 5.00mm), Sagittal T1 FS post contrast (TR: 476.00, TE: 10.00, SW: 5.00mm), Axial T2 TSE TRA FS (TR: 3820.00, TE: 49.00, SW: 5.00mm) and Axial T1 TSE TRA FS post contrast (TR: 572.00, TE: 9.00, SW: 5.00mm). Intravenous contrast: $10 \mathrm{ml}$ Dotarem. 


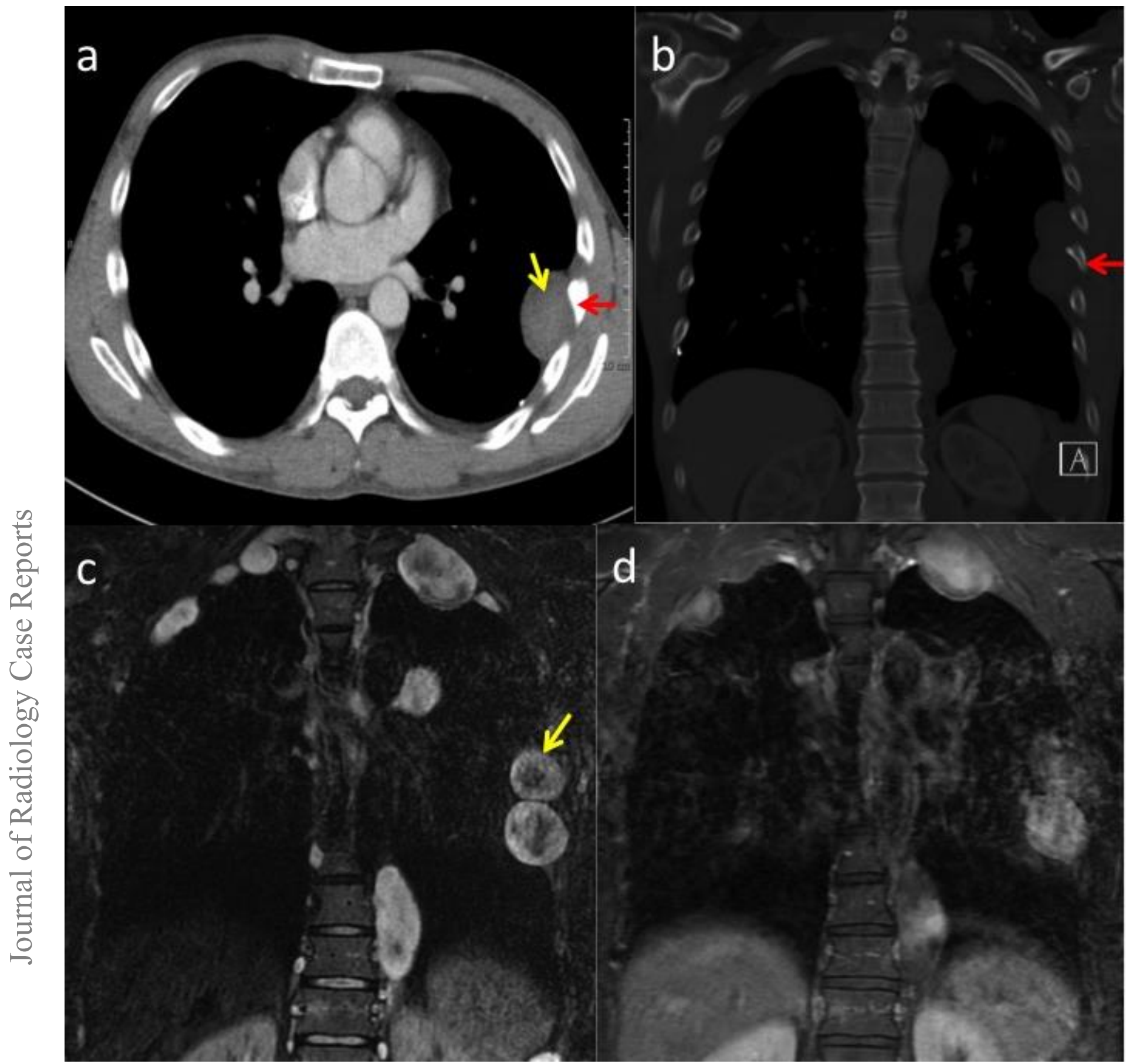

Figure 4: 41-year-old male with NF1. Multiple pleural based lesions, in keeping with multiple neurofibromas.

Findings: (a) Axial contrast-enhanced CT thorax in soft tissue window and (b) Coronal CT thorax in bony window, demonstrating a hypodense left pleural based lesion (yellow arrow) with associated rib scalloping (red arrow). (c and d) Coronal $\mathrm{T} 2$ and T1 post-contrast MRI thorax demonstrates multiple bilateral pleural based lesions, showing T2w heterogeneous signal and enhancement. A "target sign" is seen in several lesions (yellow arrow), and represents a T2 hyperintense rim surrounding a central area of low signal. Findings are in keeping with multiple neurofibromas.

Technique: (a-b): CT contrast enhanced thorax. Siemens Definition. Axial post-contrast in soft tissue window (kV: 120, mA: 161, SW: $5.00 \mathrm{~mm}$ ). Coronal post-contrast in bony window (Reformatted image, SW: 5.00mm). (c and d): MRI contrast-enhanced thorax. GE Medical Systems Signa HD 1.5T. Coronal FSE STIR (TR: 4600.00, TI: 150.00, TE: 51.22, SW: 4.00mm) and Coronal T1 FST post-contrast (TR:680.00, TE:14.83, SW: 4.00mm). Intravenous contrast: 10ml Optimark. 

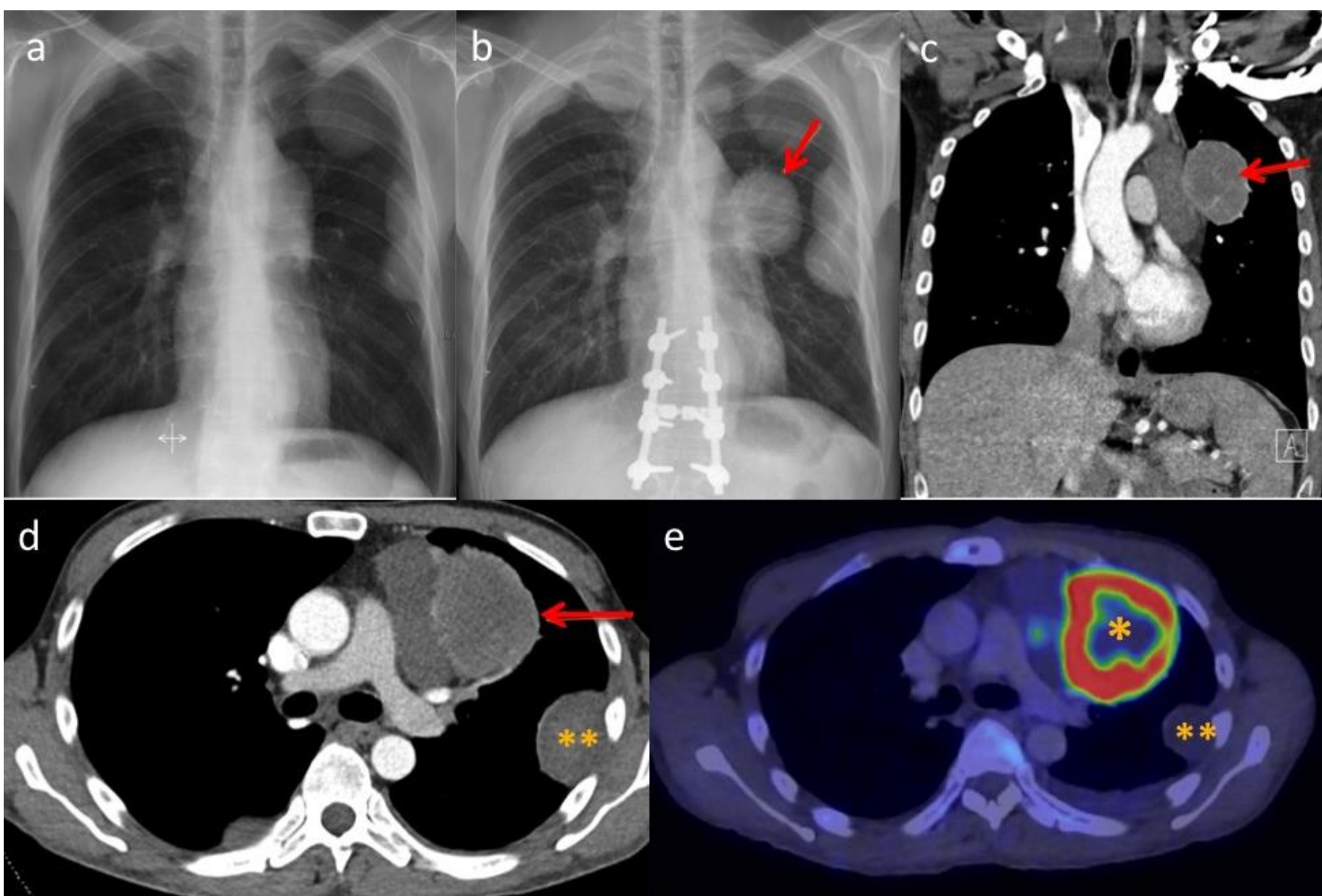

Figure 5: 41-year-old male with NF1. A new left para-mediastinal mass, demonstrating FDG avidity, suspicious for metastasis.

Findings: (a): Frontal chest radiograph (2009) shows multiple pleural based nodules, more on the left (b): Frontal chest radiograph (2019) shows a new left para-mediastinal mass (red arrow), raising suspicion for possible metastasis, in the context of newly diagnosed MPNST. Note that the patient also had interval thoracolumbar spinal instrumentation for previously known paravertebral lesions. (c) Coronal and (d) Axial contrast-enhanced CT chest demonstrates an enhancing left paramediastinal soft tissue mass, with intense FDG activity, SUV max: $24.2\left(^{*}\right)$ on PET/CT imaging (e). Note that the benign pleural based neurofibromas do not demonstrate any enhancement or FDG activity $(* *)$.

Technique: (a) Frontal chest radiograph (2009). Siemens FD-X (kVp: 109, mAs: 2.9) (b) Frontal chest radiograph (2019). Philips Medical Systems Essenta DR (kVp: 109, mAs: 2.9) (c and d): Siemens Somatom Force. Axial contrast-enhanced CT chest (kV: 100, mA: 52), Coronal contrast enhanced CT chest (Reformatted image, SW: 5.0mm). (e) PET/CT whole body fused series. GE Medical Systems DISC. Radionuclide: $7.3 \mathrm{mCi}$ of F-18 Fluorodeoxyglucose. BSL: $6.7 \mathrm{mmol} / 1$ prior to scan. Scan performed 110 minutes after IV administration of radionuclide. SW: $3.27 \mathrm{~mm}$. 


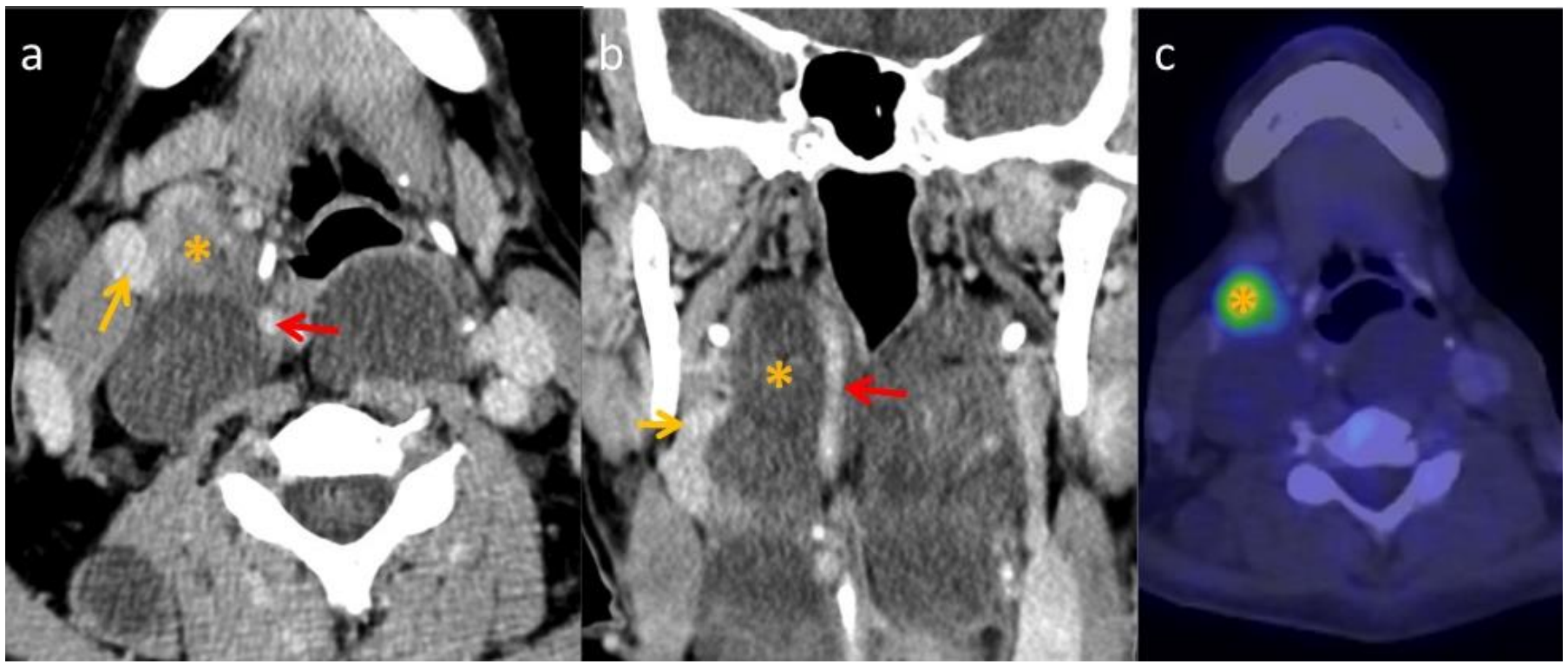

Figure 6: 41-year-old male with NF1. A right carotid body mass, likely a neurogenic tumour.

Findings: (a) Axial and (b) Coronal contrast-enhanced CT of the neck demonstrating multiple predominantly hypodense lesions in the deep spaces of the neck. Of concern, there is a solid component with heterogeneous enhancement seen in a right carotid body mass, which (c) demonstrates moderate FDG activity, SUV max: 6.3. Note the splaying of the right external (yellow arrow) and internal (red arrow) carotid arteries.

Technique: (a-b) CT neck. GE Medical Systems Lightspeed. Axial post-contrast (kV: 120, mA: 148, SW: 2.50mm) and Coronal post-contrast (Reformatted image, SW: $2.50 \mathrm{~mm}$ ).

(c) PET/CT whole body: GE Medical Systems DISC FUSED images. Radionuclide: 7.3mCi of F-18 Fluorodexoyglucose. BSL: $6.7 \mathrm{mmol} / 1$ prior to scan. Scan performed 110 minutes after IV administration of radionuclide. SW: $3.27 \mathrm{~mm}$

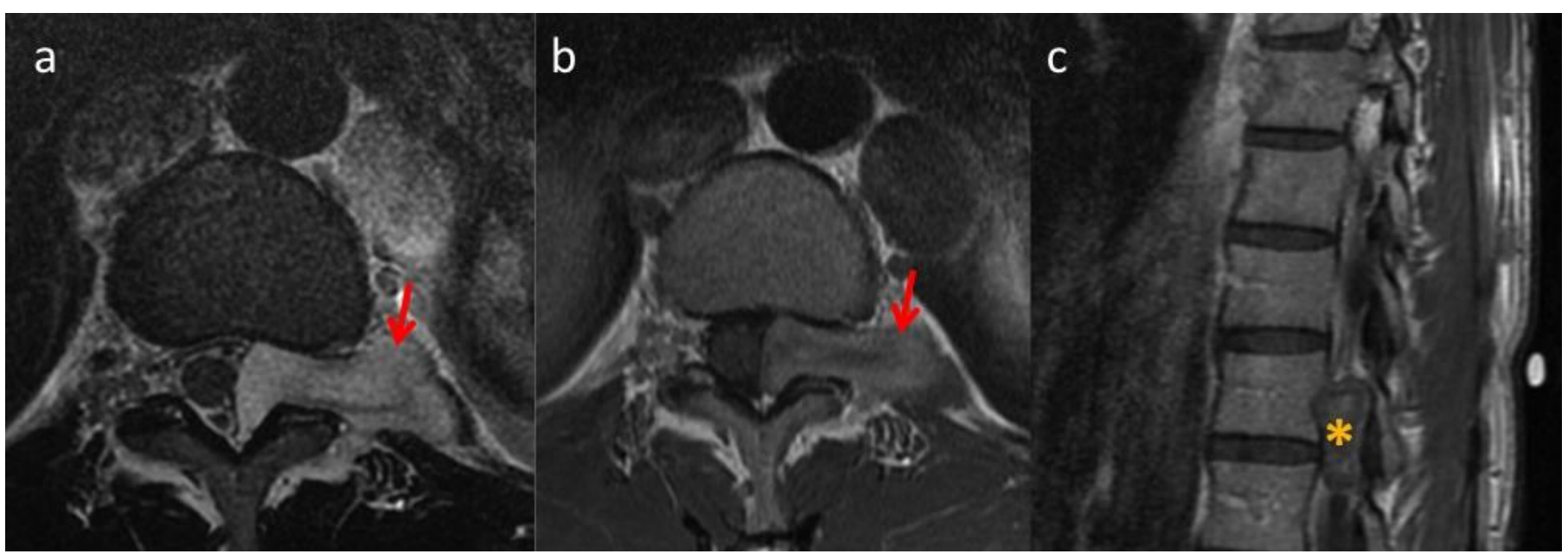

Figure 7: 41-year-old male with NF1. T11-12 paravertebral tumour.

Findings: Axial (a and b) and sagittal (c) pre and post-contrast MRI thoracic spine demonstrating a T11-12 left paravertebral "dumb-bell" tumour (arrow) causing neural foramina widening $(*)$. There is displacement for the spinal cord to the right. No abnormal cord signal detected.

Technique: (a-c) MRI contrast enhanced thoracic spine. GE medical systems Signa HD 1.5T. Axial T2 FSE (TR: 3800.00, TE: 122.02, SW: 4.00mm). Axial T1 FSE post contrast (TR: 960.00, TE:14.54, SW: 4.00mm) and Sagittal T1 FSE post contrast (TR: 980.00, TE:9.84, SW: 4.00mm). Intravenous contrast: 10ml Optimark. 


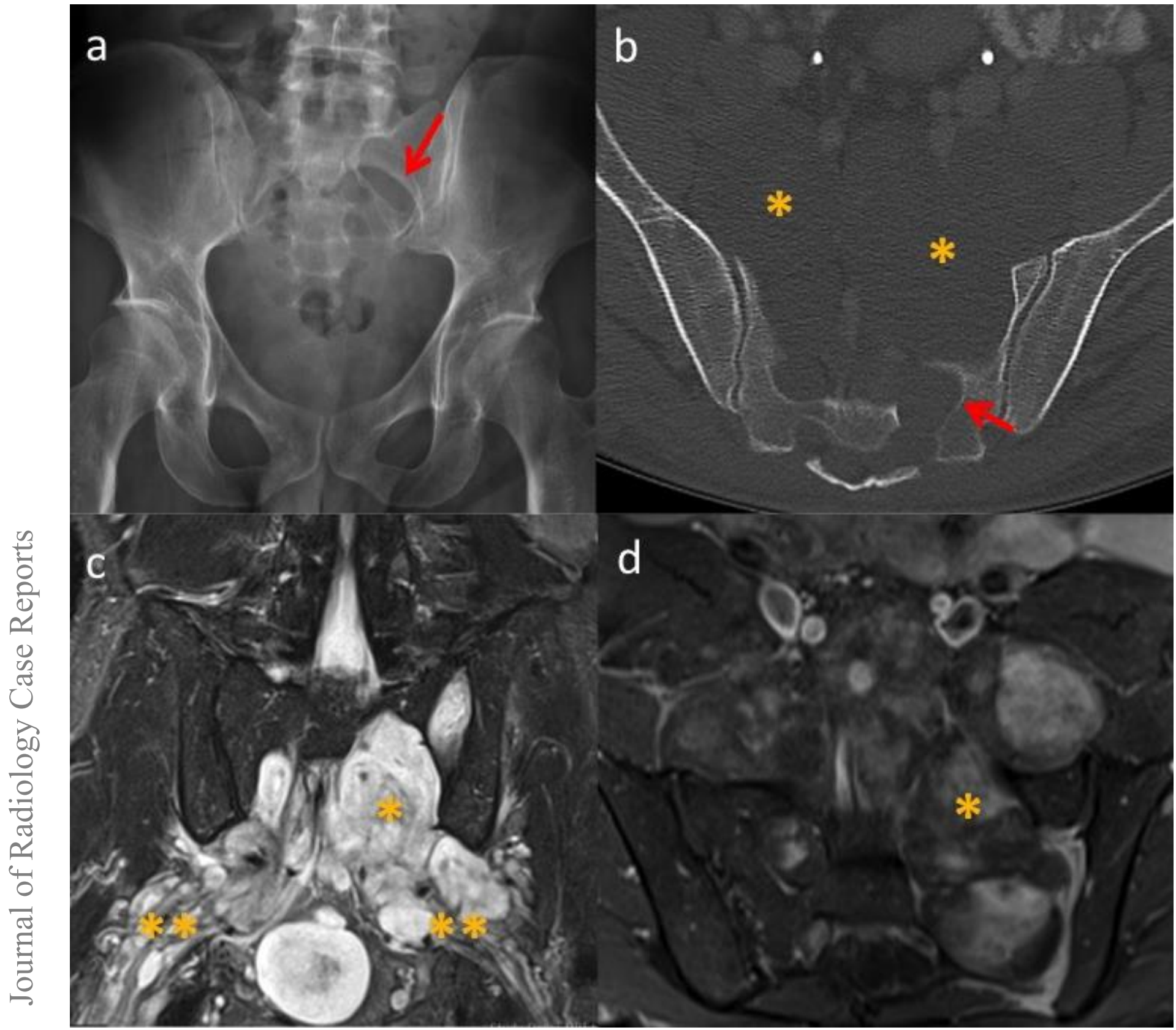

Figure 8: 41-year-old male with NF1. Multiple sacral paravertebral and lumbosacral soft tissue masses in keeping with multiple neurofibromas.

Findings: (a) Pelvic radiograph demonstrates bony scalloping and sacral foramina widening (red arrow). No pathological fracture. (b) Axial contrast enhanced CT pelvis in the bony window demonstrate multiple hypodense lobulated soft tissue lesions (*) causing sacral bony scalloping and neural foramina widening (red arrow). (c) Coronal and (d) Axial contrast enhanced MRI of the lumbosacral plexus demonstrates bilateral $\mathrm{T} 2 \mathrm{w}$ hyperintense, heterogeneously enhancing masses (*) causing widening of the sacral neural foramina, with extension to involve the bilateral lumbosacral plexus $(* *)$.

Technique: (a) Pelvic radiograph frontal projection. Siemens Fluorospot Compact FD (kVp: 81, mAs: 10). (b) Axial CT pelvis in bony windows. GE medical systems lightspeed (kV: 120, mA: 99, SW: 2.50mm). (c-d) MRI lumbosacral plexus Siemens Avanto, 1.5T, Coronal T2 TIRM (TR: 3600.00, TI: 150.0 and TE:66.00, SW: 4.00mm) and Axial T1 FS post-contrast (TR: 648.00, TE: $11.00, \mathrm{SW}: 4.00 \mathrm{~mm})$. Intravenous contrast: $5 \mathrm{ml}$ Gadovist. 


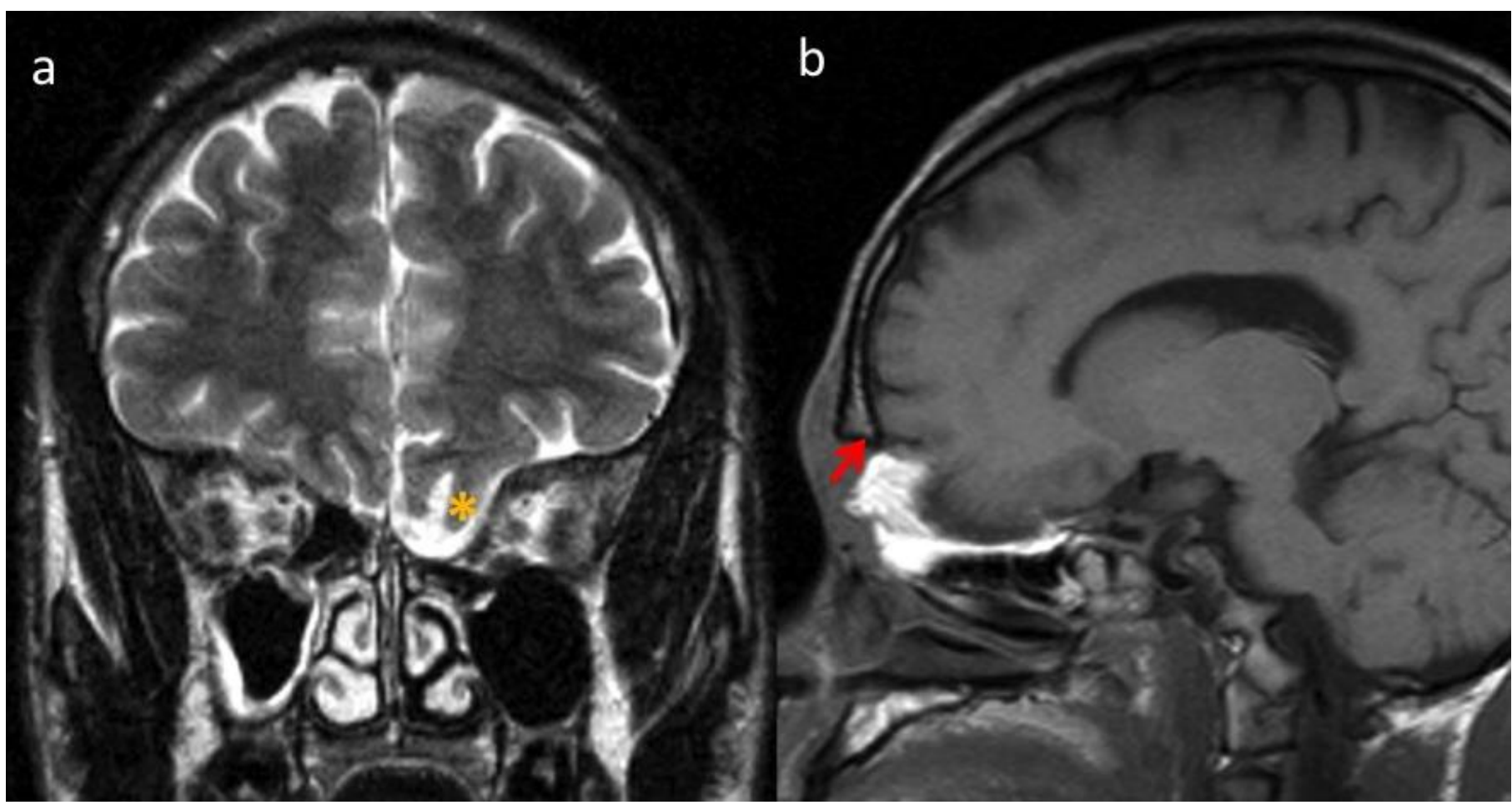

Figure 9: 41-year-old male with NF1. A left basifrontal encephalocele.

Findings: (a) Coronal T2 TSE and (b) Sagittal T1 TSE MRI brain demonstrating a left basifrontal encephalocele (*) secondary to dysplastic left basifrontal skull (red arrow).

Technique: (a-b) MRI brain: Siemens Magnetom Expert 1.0T. Coronal T2 TSE (TR: 4000.00, TE: 120.00, SW: 4.00mm) and Sagittal T1 TSE (TR: 540.00, TE: 15.00, SW: 5.00mm). 


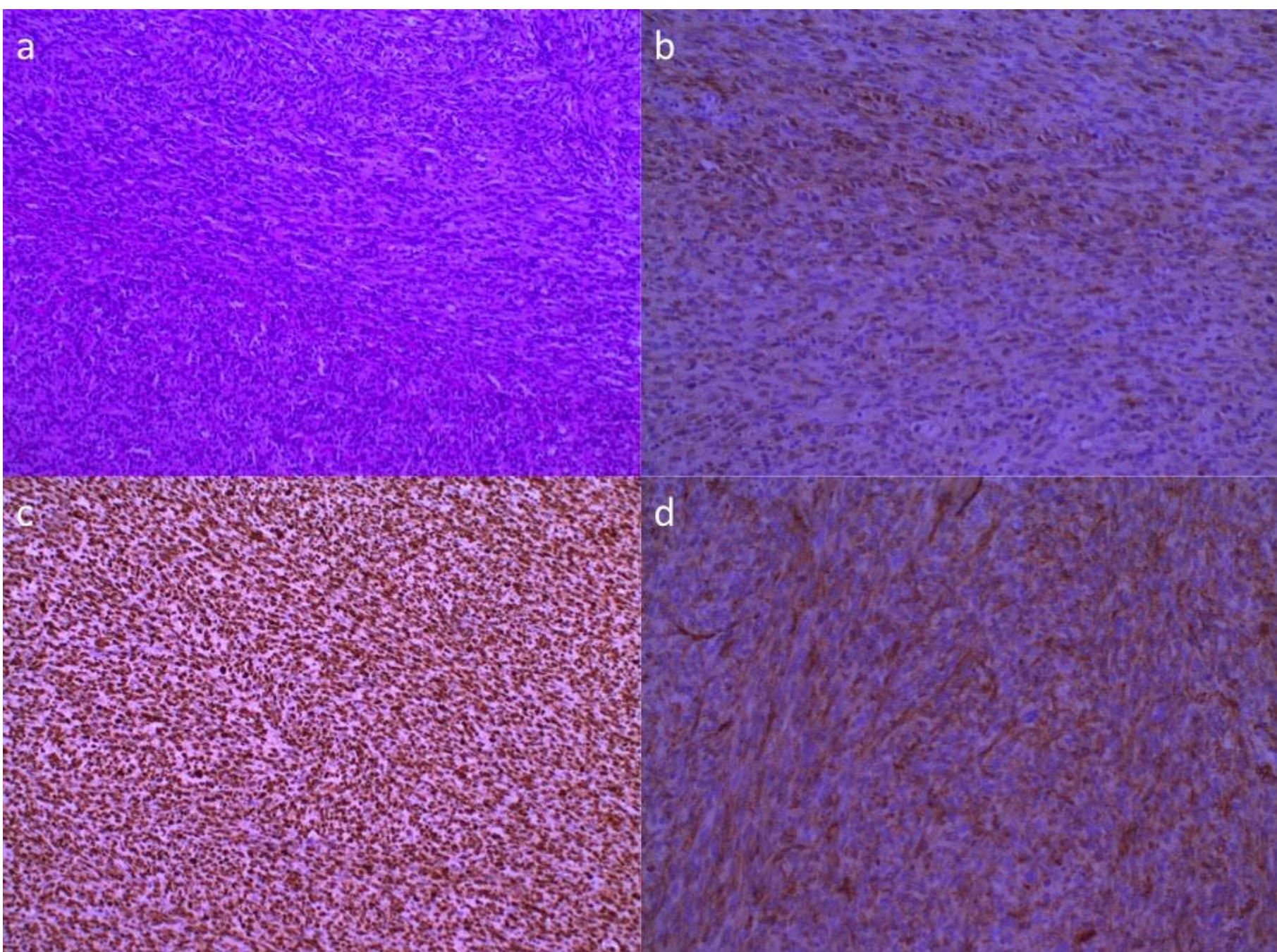

Figure 10: 41-year-old male with NF1. Histopathology of surgically excised left thigh soft tissue mass.

Surgical resection of the left proximal posterior thigh neurofibroma demonstrate a mass arising from a pre-existing plexiform neurofibroma. (a) Hematoxylin and eosin stain (magnification: 10x) shows a grade 3 malignant neurofibroma with a high mitotic rate and approximately $30 \%$ macroscopic necrosis and areas of hemorrhage. The margins are negative of tumour involvement. No evidence of lymphovascular or perineural invasion. The tumour stained positive for (b) S100 (magnification: 20x), (c) H3K27me3 (magnification: 10x) and (d) CD34 (magnification: 20x). It stained negative for SOX10, CD31 and ERG.

\begin{tabular}{|l|l|}
\hline Etiology & Malignant transformation of pre-existing nerve sheath tumour \\
\hline Incidence & $\begin{array}{l}0.001 \% \text { in the general population } \\
8-13 \% \text { amongst NF1 patients }\end{array}$ \\
\hline Gender ratio & No gender predilection \\
\hline Age predilection & Median age: 37 years \\
\hline Risk factors & $\begin{array}{l}\text { NF1 } \\
\text { Plexiform neurofibroma }\end{array}$ \\
\hline Treatment & $\begin{array}{l}\text { Surgical excision (gold standard) } \\
\text { Adjuvant/neoadjuvant chemotherapy or radiotherapy }\end{array}$ \\
\hline Prognosis & $\begin{array}{l}\text { Poor, due to high rates of local recurrence and distant metastases. } \\
\text { Disease specific mortality at 10years after definitive surgery: } 43 \%\end{array}$ \\
\hline Findings on imaging & $\begin{array}{l}\text { Increased largest dimension of mass } \\
\text { Peripheral enhancement } \\
\text { Increased heterogeneity of lesion } \\
\text { Peri-lesional oedema } \\
\text { Correlate with high-risk clinical symptoms including, new/increasing pain, new/increasing } \\
\text { radiculopathy, changes in consistency of mass (soft to hard). }\end{array}$ \\
\hline
\end{tabular}

Table 1: Summary table for malignant peripheral nerve sheath tumours. 


\begin{tabular}{|c|c|c|c|}
\hline & Radiograph & $\mathbf{C T}$ & MRI \\
\hline MPNST & $\begin{array}{l}\text { Soft tissue mass +/- bony } \\
\text { scalloping. } \\
\text { - Bony destruction, periosteal } \\
\text { reaction or pathological } \\
\text { fracture may be seen if present } \\
\text { but can be subtle. }\end{array}$ & $\begin{array}{l}\text { - Hypodense lesion with areas of } \\
\text { increased heterogeneity } \\
\text { - Increasing size } \\
\text { - Bony destruction } \\
\text { - Peri-lesional oedema }\end{array}$ & $\begin{array}{l}\text { - Increasing size } \\
\text { - Increased tumor heterogeneity } \\
\text { - Loss of target sign } \\
\text { - Peri-lesional oedema } \\
\text { - Marrow involvement }\end{array}$ \\
\hline BPNST & $\begin{array}{l}\text { Soft tissue mass +/- bony } \\
\text { scalloping/neural foramina } \\
\text { widening. }\end{array}$ & $\begin{array}{l}\text { Well-defined, smooth, round, } \\
\text { hypodense lesion +/- areas of } \\
\text { heterogeneity (usually central). }\end{array}$ & $\begin{array}{l}\text { - } \text { Target sign (specific, but not } \\
\text { sensitive) } \\
\text { - T1w hypointense } \\
\text { - } \mathrm{T} 2 \mathrm{w} \text { hyperintense }\end{array}$ \\
\hline Liposarcoma & $\begin{array}{l}\text { - Soft tissue mass with areas of } \\
\text { fatty attenuation. } \\
\text { - Bony destruction, periosteal } \\
\text { reaction, or pathological } \\
\text { fracture. }\end{array}$ & $\begin{array}{l}\text { - Mass with areas of fatty } \\
\text { attenuation. } \\
\text { - Nodular soft tissue } \\
\text { components, thick internal } \\
\text { septa. } \\
\text { - Can have fluid component. }\end{array}$ & $\begin{array}{l}\text { - Areas of T1w hyperintensity that } \\
\text { demonstrate suppression on fat } \\
\text { suppression sequences. } \\
\text { - Complex heterogeneous signal } \\
\text { with thickened septa and focal } \\
\text { nodular areas }\end{array}$ \\
\hline $\begin{array}{l}\text { Undifferentiated } \\
\text { and unclassified } \\
\text { sarcoma }\end{array}$ & $\begin{array}{l}\text { - Soft tissue mass } \\
\text { - Presence of calcifications (up } \\
\text { to } 20 \% \text { ) } \\
\text { - Bony destruction or periosteal } \\
\text { reaction }\end{array}$ & $\begin{array}{l}\text { - Presence of calcifications (up } \\
\text { to } 20 \% \text { ) } \\
\text { - Complex soft tissue mass }\end{array}$ & $\begin{array}{l}\text { - Heterogeneous complex signal } \\
\text { with no specific imaging } \\
\text { findings. }\end{array}$ \\
\hline $\begin{array}{l}\text { Fibroblastic- } \\
\text { Myofibroblastic } \\
\text { tumours }\end{array}$ & $\begin{array}{l}\text { - Soft tissue mass in the } \\
\text { cutaneous/subcutaneous plane }\end{array}$ & $\begin{array}{l}\text { - Complex solid-cystic mass } \\
\text { with necrosis and cystic } \\
\text { change }\end{array}$ & $\begin{array}{l}\text { Complex solid-cystic mass with } \\
\text { areas of necrosis and ill-defined } \\
\text { infiltrative margins. } \\
\text { - Areas of T2/T1w hypointensities } \\
\text { correspond to fibrous component. }\end{array}$ \\
\hline & Contrast enhancement & US & PET \\
\hline MPNST & $\begin{array}{l}\text { - Peripheral enhancement } \\
\text { - Heterogeneous }\end{array}$ & Of limited value & $\begin{array}{l}\text { - Avid FDG uptake (non-specific), } \\
\text { may help to differentiate between } \\
\text { malignant and benign features } \\
\text { - Identifies metastases }\end{array}$ \\
\hline BPNST & $\begin{array}{l}\text { - Central enhancement } \\
\text { - Homogeneous }\end{array}$ & Of limited value & $\begin{array}{l}\text { Mild-moderate FDG uptake in solid } \\
\text { components }\end{array}$ \\
\hline Liposarcoma & Heterogeneous enhancement & Of limited value & Avid FDG uptake \\
\hline $\begin{array}{l}\text { Undifferentiated } \\
\text { and unclassified } \\
\text { sarcoma }\end{array}$ & Heterogeneous enhancement & Of limited value & Avid FDG uptake \\
\hline $\begin{array}{l}\text { Fibroblastic- } \\
\text { Myofibroblastic } \\
\text { tumours }\end{array}$ & $\begin{array}{l}\text { Heterogeneous enhancement } \\
\text { with necrotic/cystic areas }\end{array}$ & Of limited value & $\begin{array}{l}\text { Avid FDG uptake in the solid } \\
\text { components }\end{array}$ \\
\hline
\end{tabular}

Table 2: Differential diagnosis table for malignant peripheral nerve sheath tumours.

\section{ABBREVIATIONS}

BPNST $=$ Benign peripheral nerve sheath tumour

$\mathrm{CT}=$ Computed Tomography

FDG = Flurodeoxyglucose

MPNST $=$ Malignant peripheral nerve sheath tumours

MRI = Magnetic Resonance Imaging

NF1 $=$ Neurofibromatosis type 1

US = Ultrasonography

\section{KEYWORDS}

Malignant peripheral nerve sheath tumour; MPNST; neurofibrosarcoma; neurofibromatosis type 1; NF1; plexiform neurofibroma; malignant transformation; MRI; CT; PET

\section{Online access}

This publication is online available at: www.radiologycases.com/index.php/radiologycases/article/view/4028

\section{Peer discussion}

Discuss this manuscript in our protected discussion forum at: www.radiolopolis.com/forums/JRCR

\section{Interactivity}

This publication is available as an interactive article with scroll, window/level, magnify and more features. Available online at www.RadiologyCases.com 